\title{
Note sur le rôle vecteur des Ardeides dans certaines helminthoses
}

\author{
par P. DAYNES $(*)$ et J. BLANCOU $(* *)$
}

\begin{abstract}
RESUME
Des œufs de Fasciola gigantica et Ascaris suum peuvent rester viables plus de 48 heures dans l'intestin de certains Ardeidae. Ardeola idae, espèce migratrice d'Afrique à Madagascar, pourrait donc théoriquement participer au transfert d'helminthoses correspondantes à plus de 1.000 kilomètres.
\end{abstract}

La propagation des helminthoses est, en règle générale, assurée par l'arrivée d'hôtes parasités en régions indemnes où les formes infestantes excrétées reconstituent leur cycle.

Toutefois ces mouvements sont souvent limités par des obstacles géographiques et, en ce qui concerne les animaux domestiques, par l'application des règlements sanitaires (1).

Le but de cette étude est d'apprécier les possibilités d'extension des helminthoses, malgré ces obstacles, lors de l'intervention d'espèces aviennes sauvages: les ardéïdés.

\section{MATERIEL}

Deux espèces d'ardéïdés ont été étudiées quant à leur rôle vecteur dans deux helminthoses majeures à Madagascar : la fasciolose et l'ascaridose (2) (3).

\section{ARDEIDES}

Cinq individus de chacune des deux espèces suivantes ont été utilisés :

(*) I.E.M.V.T. Service de Parasitologie. B.P. 1053. Addis-Abeba. Ethiopie.

$\left({ }^{* *}\right)$ I.E.M.V.T. Service de Bactériologie. B.P. 862, Tananarive. Madagasear.
1. Ardeola idae (Hartlaub), le crabier malgache

Cette espèce ne s'éloigne guère des marécages, où elle trouve sa nourriture principale. Ces marécages constituent pour la plupart l'abreuvoir naturel des animaux domestiques et ils sont l'objet d'une contamination fécale constante, comme nous avons pu nous en assurer par analyse bactériologique: les oiseaux qui y vivent sont donc exposés à ingérer fréquemment les cufs de parasites excrétés par leurs hôtes. La majeure partie de la population de crabier malgache migre en Afrique Orientale de mai à octobre (4).

2. Bubulcus ibis (Linné), le héron garde bœuf

Cette espèce peut vivre très éloignée du milieu aquatique car elle se nourrit surtout d'insectes et en particulier de ceux dérangés par les troupeaux domestiques ou attirés par les excréments de ces animaux. Cette espèce est donc exposée, plus encore que la précédente, à ingérer des cufs de parasites. Elle effectue des migrations à l'intérieur de l'Ile.

\section{HELMINTHES}

Deux espèces ont été étudiées: Fasciola gigantica (Cobbold, 1855) et Ascaris suum (Goeze, 1782). 


\section{METHODES}

Les oiseaux sauyages, capturés dans la région de Tananarive et destinés à l'expérience, sont soumis à une observation de 8 jours au cours de laquelle leurs excréments sont soumis à un examen microscopique quotidien. La plupart d'entre eux souffrant d'un parasitisme naturel par des nématodes et des trématodes (strigéidés), l'absence totale d'œufs de Fasciola gigantica et d'Ascaris suum doit être contrôlée par mensurations de tous les œufs révélés par cette coproscopie. Ce n'est que lorsque l'observation préalable de 8 jours révèle l'absence totale d'cufs de l'helminthe étudié que les oiseaux sont soumis à une ingestion forcée d'œufs de cette espèce. Pour cela, les œufs sont récoltés dans la journée sur des parasites obtenus à l'abattoir, dénombrés, et déposés de force dans l'œsophage après introduction dans une capsule de gélatine.

\section{RESULTATS}

Les résultats sont de deux ordres, pour chaque espèce avienne et chaque espèce d'helminthes:

- Durée de séjour des cufs dans le tube digestif de l'oiseau (cette durée étant, en moyenne, de 30 heures pour une particule colorée inerte);

- Vitalité des œufs après ce séjour dans le tube digestif.

\section{Ardeola idae}

\section{Fasciola gigantica}

- Après l'ingestion forcée de 3.000 aufs $( \pm 200)$ le record de séjour dans le tractus digestif est de 60 heures.

- La vitalité de ces œufs, appréciée après incubation en chambre humide, reste entière après ce séjour dans l'intestin des oiseaux. Toutefois, dans certains cas, l'embryogenèse est arrêtée aux premiers stades et l'œuf n'éclôt pas.

\section{Bubulcus ibis}

\section{Fasciola gigantica}

- Après l'ingestion forcée de $3.000( \pm 200$ ) le record de séjour dans le tractus digestif est de 60 heures. Si l'ingestat est plus important (30.000 cufs) la durée du séjour peut s'élever jusqu'à quatre jours.

- La vitalité des aufs, appréciée comme précédemment, est conservée même après un séjour de plus de 48 heures.

\section{Ascaris suum}

- Après l'ingestion forcée de 14.000 cufs ( \pm 700$)$ le record de séjour des œufs dans l'intestin s'élève à neuf jours.

- La vitalité peut être démontrée sur ces œufs après un séjour de 48 heures dans l'intestin, mais pas au-delà (dans nos condjtions expérimentales).

\section{CONCLUSION - DISCUSSION}

Sur le plan théorique, le transport des cufs de Fasciola et Ascaris est possible sans altération de leur vitalité pendant 48 heures au moins, temps durant lequel l'oiseau porteur de ces œufs peut parcourir (« en vitesse de croisière ») plus de mille kilomètres (4). Ardeola idae, en particulier, espèce migratrice, pourrait donc transférer ces helminthoses d'Afrique Orientale sur l'ensemble du territoire malgache. En pratique, toutefois, ces transferts sont certainement limités par la sédentarité des oiseaux durant la majeure partie de l'année, la faible quantité d'œufs ingérés, et les rares opportunités laissées aux formes écloses de reconstituer leur cycle.

\section{Remerciement}

Nous remercions vivement MM. R. ALBIGNAC et G. RANDRIANASOLO qui nous ont aimablement fourni les oiseaux nécessaires à ces expériences au Parc de l'O.R.S.T.O.M. 


\section{SUMMARY}

\section{Ardeidae as vector of certain helminth disease}

Eggs of Fasciola gigantica and Ascaris sum are still alive 48 hours after their ingestion by some Ardeidae. Ardeola idae, a migratory species from Africa, could theoretically transfer these disease in Madagascar on more than 1.000 kilometers.

\section{RESUMEN}

Nota sobre el papel vector de los Ardeidae en ciertas helmintosis

Huevos de Fasciola gigantica y Ascaris suum pueden seguir viviendo más de 48 horas en el intestino de ciertos Ardeidae. Pués, Ardeola idae, especie de paso de Africa hacia Madagascar, podria teoricamente participar a la transferencia de helmintosis correspondientes hasta más de 1000 kilometros.

\section{BIBLIOGRAPHIE}

1. BLANCOU (J.). Interractions pathologiques entre mammifères et oiseaux domestiques et sauvages à Madagascar. Conf. int. pour l'utilisation rationnelle et la conservation de la nature, Tananarive 1970, Doc. no D 13.

2. DAYNES (P.). Note sur les helminthoses des ani- maux domestiques reconnues à Madagascar. Rev. Elev. Méd. vét. Pays trop. 1964, 17 (3) : 477-490.

3. DAYNES (P.). La distomatose à Madagascar. Cycle de Fasciola gigantica. Rev. Elev. Méd. vét. Pays trop. 1967, 20 (4) : 557-562.

4. DORST (J.). Les migrations des oiseaux. Paris, Payot, 1950. 